\title{
The floor in the solar wind: status report
}

\author{
E. W. Cliver ${ }^{1}$ \\ ${ }^{1}$ Space Vehicles Directorate, Air Force Research Laboratory Sunspot, NM, USA \\ email: ecliver@nso.edu
}

\begin{abstract}
Cliver \& Ling (2010) recently suggested that the solar wind had a floor or groundstate magnetic field strength at Earth of $\sim 2.8 \mathrm{nT}$ and that the source of the field was the slow solar wind. This picture has recently been given impetus by the evidence presented by Schrijver et al. (2011) that the Sun has a minimal magnetic state that was approached globally in 2009, a year in which Earth was imbedded in slow solar wind $\sim 70 \%$ of the time. A precursor relation between the solar dipole field strength at solar minimum and the peak sunspot number $\left(S S N_{M A X}\right)$ of the subsequent 11-yr cycle suggests that during Maunder-type minima (when $S S N_{M A X}$ was $\sim 0$ ), the solar polar field strength approaches zero - indicating weak or absent polar coronal holes and an increase to nearly $\sim 100 \%$ in the time that Earth spends in slow solar wind.
\end{abstract}

Keywords. Sun, magnetic fields, solar wind

\section{Introduction}

Does the solar wind, at the current stage of the Sun's life, have a ground state or floor? Was such a ground state approached during the Maunder Minimum? Interest in the notion of such a floor has increased because of the recent prolonged and deep solar minimum and the prospect that we might be entering a Gleissberg-type minimum such as that of $\sim 1900$ or perhaps even a Maunder-type minimum (Eddy, 1976).

The concept of a floor in the solar wind magnetic field strength $(B)$ was introduced separately by Svalgaard and Cliver (2007) and Owens et al. (2008). Svalgaard and Cliver posited a floor in $B$ of $\sim 4.6 \mathrm{nT}$ based on their long-term geomagnetic-based reconstruction of $B$ and the correlation of $B$ with the sunspot number $(S S N)$ while Owens et al. (2008) obtained a $B$ value of $\sim 4 \mathrm{nT}$ at solar minimum when the rotation-averaged coronal mass ejection rate was extrapolated to zero. Both papers viewed the floor as the state of the near-Earth solar wind in the absence of 11-yr cyclic activity.

Shortly after these floor values were proposed, the solar wind $B$ dropped to its lowest values observed during the space age, $\sim 4.2 \mathrm{nT}$ in 2008 and $\sim 3.9 \mathrm{nT}$ in 2009, prompting downward revisions of the proposed levels by both groups of authors: to $\sim 3.7 \mathrm{nT}$ by Crooker and Owens (2010) and to $\sim 2.8 \mathrm{nT}$ by Cliver and Ling (2010). Here I will focus on the work by Cliver and Ling (2010) which also suggested a novel source for such a ground state - the omnipresent slow solar wind. The chain of reasoning that led to this suggestion began with the paper on sunspot cycle prediction by Schatten et al. (1978).

\section{Precursor relations and the floor}

Schatten et al. (1978) argued that because (in the dynamo model for solar activity) poloidal fields at solar minimum provide the "seed" for the toroidal fields of the subsequent maximum, the peak sunspot number of a cycle should be related to the strength of the solar polar fields at the preceding minimum. It was difficult to test this idea directly at the time however, because reliable measurements of the solar polar fields had 
been made at only one solar minimum up to that point. A direct use of this method (i.e., not based on proxy input parameters such as geomagnetic indices, e.g., Wang and Sheeley, 2009) was made by Svalgaard et al. (2005) who predicted that the present cycle would be the smallest in $\sim 100$ years with a peak sunspot number $\left(S S N_{M A X}\right)$ of $75 \pm 8$. This prediction (Fig. 1(a)), which was based on only two cycles and a force fit through the origin, was close to the value of 90 eventually adopted by the NOAA/NASA/ISES prediction panel (http://www.swpc.noaa.gov/SolarCycle/SC24/index.html).

Because the floor of $\sim 4.6 \mathrm{nT}$ in the solar wind proposed by Svalgaard and Cliver (2007) was thought to apply to the Maunder Minimum, the precursor relation in Fig. 1(a) implies that this value, which was approached at every 11-yr minimum during the space age, is relatively independent of the solar polar field strength. This misconception was based in part on Ulysses observations through November 2006 (Balogh \& Smith, 2006) which showed little change in the radial field strength between fast latitude scans near the minima following solar cycles 22 and 23. As Svalgaard and Cliver (2007) wrote, "While the radial IMF strengths $\left[B_{R}\right]$ are essentially identical for these minima, the solar polar magnetic fields are $\sim 40 \%$ weaker at present $(60 \mu \mathrm{T}$ vs. $100 \mu \mathrm{T}$ for $\sim 1995$; Svalgaard et al. (2005) and current data from Wilcox Solar Observatory [WSO]). It seems hard to escape the conclusion that the polar fields do not determine the magnitude of the IMF at solar minimum." Subsequently, Smith and Balogh (2008) reported that $B_{R}$ measured by Ulysses dropped by $\sim 35 \%$ between the minima following cycles 22 and 23 and, as noted above, $B$ dropped below $4 \mathrm{nT}$ in the ecliptic plane in 2009. Clearly solar wind $B$ was responding to the change in the solar polar field strength. The relationship between solar minimum values of the solar dipole moment $[D M]$ and near-Earth $B$ for the last four minima can be seen in Fig. 2 (taken from Cliver and Ling, 2010). Linear extrapolation of the regression line indicates that when the Sun's dipole moment goes to zero [implying from Fig. 1(a) that the subsequent solar cycle will have a $S S N_{M A X}$ value $\sim 0$ (i.e., Maunder-type minimum conditions; Eddy, 1976, Hoyt and Schatten, 1998)], the solar wind will have a floor of $\sim 2.6 \mathrm{nT}$.
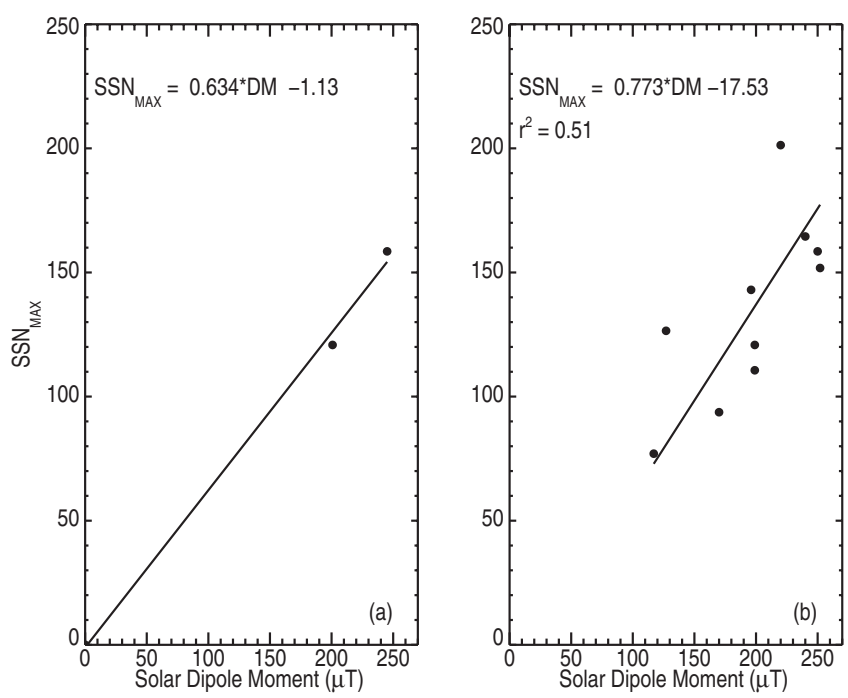

Figure 1. (a) Precursor relation used by Svalgaard et al. (2005) to predict that cycle 24 would be the smallest in $\sim 100$ years with a peak sunspot number of $\sim 75$. (b) Same as (a) but with more data points based on the correlation between $B_{M I N}$ and $D M$ in Fig. 2 and a long-term reconstruction of solar wind $B$ (Svalgaard \& Cliver, 2010) 


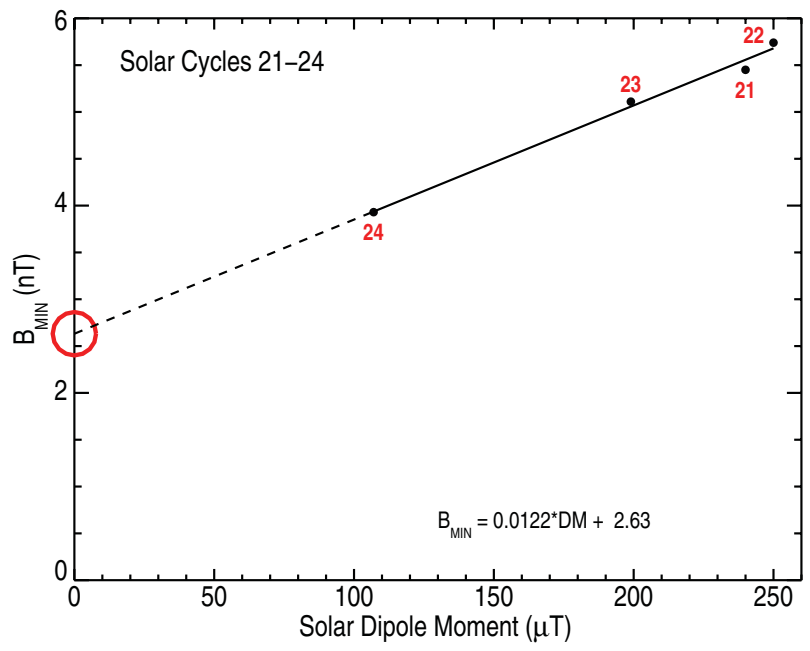

Figure 2. Solar magnetic field strength $\left(B_{M I N}\right)$ at the last four solar minima (cycles 21-24) plotted vs. the solar dipole-field strength. The linear extrapolation suggests the existence of a floor in $B_{M I N}$ at a value of $\sim 2.6 \mathrm{nT}$ (red circle, see the online version of this paper) (from Cliver and Ling, 2010).

The relationship between $B_{M I N}$ and $D M$, in combination with the long-term geomagnetic-based reconstruction of solar wind $B$ (Svalgaard and Cliver, 2010), can be used to infer $D M$ going back in time. Thus it is possible to substantiate (Fig. 1(b)) the prediction of Svalgaard et al. that was based on only two cycles. For an input of $119.3 \mu \mathrm{T}$ (Svalgaard et al., 2005), the equations in (a) and (b) both give a prediction of $\sim 75$. Note that for zero $S S N_{M A X}$, the dipole moment is zero within the uncertainty $(23 \pm 32 \mu \mathrm{T})$ justifying the fit through the origin in Fig. 1(a). Alternatively, one could use $B_{M I N}$ as a proxy for $D M$ in a precursor relationship, as was done by Cliver and Ling (2010), who found that a peak sunspot number of $\sim 0$ corresponded to a floor in $B$ of $\sim 2.9 \mathrm{nT}$, near the value of $\sim 2.6 \mathrm{nT}$ inferred from Fig. 2. Cliver and Ling therefore suggested that the floor had a value of $\sim 2.8 \mathrm{nT}$, significantly below the Svalgaard et al. (2007) value of $\sim 4.6 \mathrm{nT}$ and also under Crooker and Owens' revised value of $\sim 3.7 \mathrm{nT}$. The reduced floor value of $2.8 \mathrm{nT}$ has several advantages. It permits the predicted $S S N_{M A X}$ values to go to $\sim 0$ in a precursor relation based on $B_{M I N}$. It makes the $\sim 50 \%$ drop in solar wind $B_{M I N}$ (measured above the floor) between the minima preceding cycles 23 and 24 comparable to the corresponding $\sim 45 \%$ drop in $D M$ (Cliver \& Ling, 2010). Finally, a floor at $\sim 2.8 \mathrm{nT}$ accommodates most of the 10,000 year ${ }^{10}$ Be-based reconstruction of $B$ from Steinhilber et al. (2010) except for several sharp dropouts where $B$ goes to $\sim 0 \mathrm{nT}$ (Fig. 3).

\section{Is the slow solar wind the source of the floor?}

Cliver and Ling (2010) noticed that the revised floor value of $\sim 2.8 \mathrm{nT}$ corresponded roughly to the relatively constant contribution of the slow solar wind to annual averages of $B$ (Fig. 4, taken from Cliver and Ling, 2010). They attributed the fact that the contribution of the slow solar wind was generally less than $2.8 \mathrm{nT}$ to the accounting scheme of Richardson et al. $(2000,2002)$ which was designed to apportion geomagnetic activity to the three basic wind types [coronal mass ejections (CMEs), high-speed streams (HSSs), and slow solar wind (SSW)]. In this scheme, co-rotating interaction regions and shocksheath regions are attributed to HSSs and CMEs, respectively. At solar minimum, when 
CMEs essentially disappear and high-stream speeds are less frequent, a more accurate accounting of the SSW contribution can be obtained. During the magnetic minimum year of 2009, Earth was imbedded in SSW $\sim 70 \%$ of the time and extrapolations of $B$ to a value of $2.8 \mathrm{nT}$ indicate that Earth would be immersed in SSW, with a speed of $\sim 300 \mathrm{~km} \mathrm{~s}^{-1}$, over $90 \%$ of the time at the floor (Cliver \& Ling, 2010). In other words, the slow solar wind is the floor.

\section{Is the "minimal solar activity" identified by Schrijver et al. the source of the slow solar wind and the floor?}

Cliver and Ling (2010) proposed that "the floor corresponds to a baseline (non-cyclic or ground state) open solar flux of $\sim 8 \times 10^{13} \mathrm{~Wb}$ which originates in persistent small-scale (supergranular or granular) field." Recently, Schrijver et al. (2011), from an analysis of a series of Ca II K line data taken at Kitt Peak from 1974-present for the quietest regions on the solar disk, reported "a baseline activity level that is independent of the global sunspot cycle ... regularly observed locally in the quiet-Sun network", i.e., a floor in solar magnetic activity. Quoting from their abstract, "We argue that there is a minimum state of solar magnetic activity associated with a population of relatively small magnetic bipoles which persists even when sunspots are absent ... The minimal solar activity ... was approached globally after an unusually long lull in sunspot activity in 2008-2009. Therefore, the best estimate of magnetic activity ... for the least-active Maunder Minimum phases appears to be provided by direct measurement in 2008-2009." The existence of such a floor in solar magnetic activity is a requirement for a floor in the solar wind. Moreover, the suggestion that 2009 provided our best glimpse thus far of the magnetic state of the Sun during the Maunder Minimum is consistent with the notion that the solar wind during the Maunder Minimum was characterized by slow solar wind. If, in fact, the precursor relation in Fig. 1 extends down to $D M=0\left(S S N_{M A X} \sim 0\right)$, then we would expect the polar coronal holes and high-speed stream component of the solar wind (in which Earth was imbedded for $\sim 25 \%$ of the time during 2009) to disappear during Maunder-type minima, leaving only slow solar wind.

Schrijver et al. (2011) obtained a minimum total unsigned solar flux of $15 \times 10^{22} \mathrm{Mx}$ from SOHO MDI (Scherrer et al., 1995) observations during 2009. Approximately $5 \%$ of

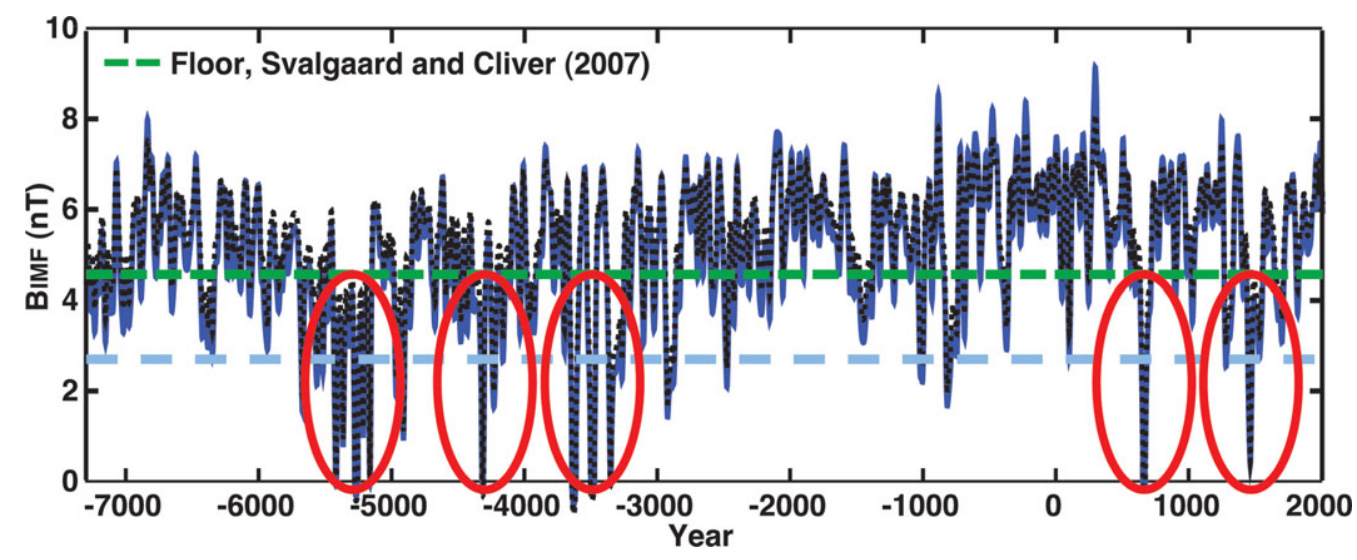

Figure 3. ${ }^{10}$ Be-based reconstruction of 9,300 years of solar wind $B$ adapted from Steinhilber et al. (2010). The positions of the old (dashed green line) and new (dashed blue line) floors are shown. The red ovals encompass the sharp dropouts to $\sim 0 \mathrm{nT}$. The $B$ data are 40 -yr running means. For color figures see the online version of this paper. 


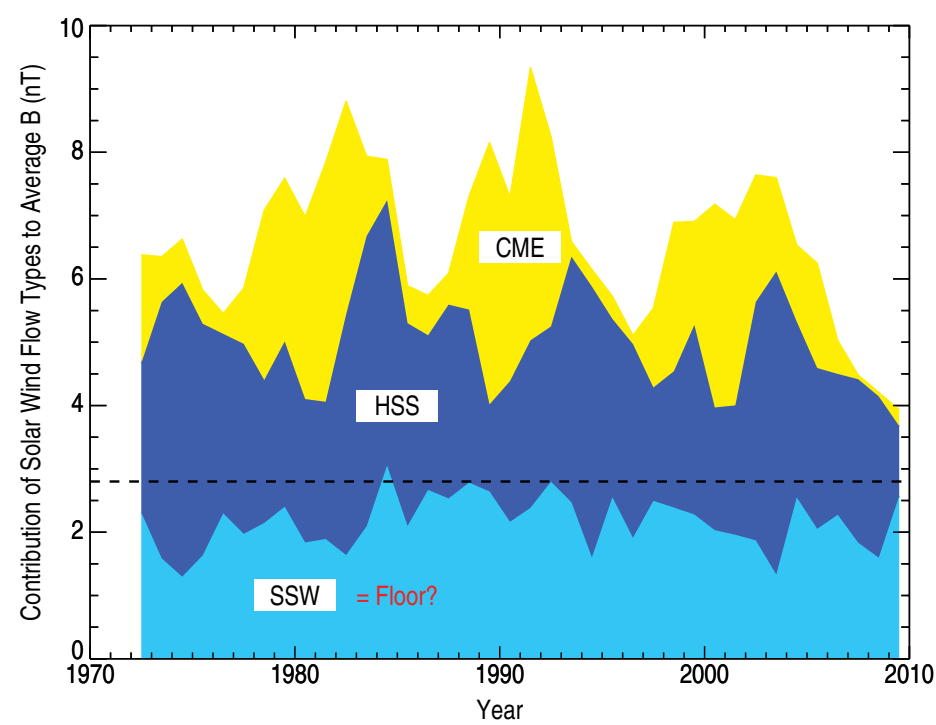

Figure 4. Cumulative distribution of the contributions of SSW, HSSs, and CMEs to average $B$ from 1972-2009. The dashed line is drawn at the floor value of $\sim 2.8 \mathrm{nT}$ (from Cliver and Ling, 2010). See color figure in the online version of this paper.

this flux would need to escape the Sun to account for the floor in $B$ deduced by Cliver and Ling (2010).

\section{Questions}

Open questions abound. Does the "Livingston-Penn" effect (a secular decrease in the maximum magnetic field strength of sunspots; Livingston \& Penn, 2009, and references therein) change the nature of spots at extended minima, thereby affecting the linear extrapolation in the precursor relation between $S S N_{M A X}$ and $D M$ or $B_{M I N}$ ? Did the solar cycle continue during the Maunder Minimum at a time when Fig. 1 suggests that the solar dipole field essentially disappeared? The concentration of ${ }^{10} \mathrm{Be}$ in ice cores suggests that it did (Beer et al., 1998; Berggren et al., 2009), while the auroral record (Siscoe, 1980) indicates a general diminution in solar wind activity during this time. Can the "minimal solar activity" of Schrijver et al. (2011) maintain a solar wind in the absence of the solar polar fields and, if so, would that solar wind be primarily slow solar wind? When the Maunder Minimum was rediscovered, both Parker (1975) and Eddy (1976) hypothesized that the Sun would resemble a giant coronal hole with high speed wind in all directions. The most recent minimum, which presumably has given us our best glimpse of Maunder Minimum conditions thus far, suggests quite the opposite.

\section{Acknowledgements}

We thank Sarah Gibson, Cristina Mandrini, Hebe Cremades, and David Webb for organizing a very timely and stimulating conference.

\section{References}

Balogh, A. \& Smith, E. J. 2006, AGU Fall Meeting, Abstract No. SH44A-05

Beer, J., Tobias, S., \& Weiss, N. 1998, Solar Phys. 181, 237 
Berggren, A.-M., et al. 2009, Geophys. Res. Lett. 36, L11801

Cliver, E. W. \& Ling, A. G. 2010, Solar Phys. DOI: 10.1007/s11207-010-9657

Crooker, N. U. \& Owens, M. J. 2010, in: Cranmer, S., Hoeksema, T., \& Kohl, J. (eds.) Proc. of SOHO 23: Understanding a Peculiar Minimum CS-428 (San Francisco: ASP), p. 279

Eddy, J. A. 1976, Science 192, 1189

Hoyt, D. V. \& Schatten, K. H. 1998, Solar Phys. 181, 491

Livingston, W. \& Penn, M. 2009, Eos, Trans. Am. Geophys. Union 90, 257

Owens, M. J., et al. 2008, Geophys. Res. Lett. 35, L20108

Parker, E. N. 1975, Sci. Am. 233, 42

Richardson, I. G., Cliver, E. W., \& Cane, H. V. 2000, J. Geophys. Res. 105, 18203

Richardson, I. G., Cane, H. V., \& Cliver, E. W. 2002, J. Geophys. Res. 107, 1187

Schatten, K. H., Scherrer, P. H., Svalgaard, L., \& Wilcox, J. M. 1978, Geophys. Res. Lett. 5, 411

Scherrer, P. H., et al. 1995 Solar Phys. 162, 129

Schrijver, C. J., Livingston, W. C., Woods, T. N., \& Mewaldt, R. A. 2011, Geophys. Res. Lett. 38, L06701

Siscoe, G. L. 1980, Rev. Geophys. Space Phys. 18, 647

Smith, E. J. \& Balogh, A. 2008, Geophys. Res. Lett. 35, L22103

Steinhilber, F., Abreu, J. A., Beer, J., \& McCracken, K. G. 2010, J. Geophys. Res. 115, A01104

Svalgaard, L., Cliver, E. W., \& Kamide, Y. 2005, Geophys. Res. Lett. 32, L01014

Svalgaard, L. \& Cliver, E. W. 2007, ApJ (Lett.) 661, L203

Svalgaard, L. \& Cliver, E. W. 2010, J. Geophys. Res. 115, A09111

Wang, Y.-M. \& Sheeley, N. R., Jr. 2009, ApJ (Lett.) 649, L11

\section{Discussion}

Arnab Choudhuri: In our paper of 2007 (Jiang et al., MNRAS, 381, 1527) we made some effort to use the polar faculae as a proxy for the next activity cycle. We found two outliers. We would be happy to discuss this with you.

ED Cliver: Yes, of course. Comparison of Jiang et al. (2007) with Figure 1(b) revealed that the two outliers based on polar faculae (for the minima preceding Cycles 16 and 20) were not the same as for ours (Cycles 15 and 19). While the outliers in the Jiang et al. study would have resulted in over-predictions, the outliers in ours corresponded to under-predictions of the cycle peak SSN.

SACHA BRUN: Why do you extrapolate between the floor IMF and the sunspot number, as we expect the Sun to drive a dynamo field independent of sunspot emergence, one may want to look instead at the polar field during quiet phases?

ED Cliver: That comparison/extrapolation is shown in Figure 2 and indicates a solar wind Bmin value of $\sim 2.6 \mathrm{nT}$ when the solar polar field strength approaches zero at solar minimum. 